\title{
Testing the Validity of Conditional Arguments Using Physical Models
}

\section{Ron LEONARD}

Department of Philosophy, University of Nevada, Las Vegas 4505 Maryland Parkway, Box 455028, , Las Vegas, Nevada 89154-5028

U.S.A.

E-mail: pleroma@mindspring.com

.In teaching critical reasoning it is often helpful for students to visualize logical relationships by employing diagrams of simple analogous physical situations that they already understand. Testing the validity of arguments composed of categorical propositions by using Venn diagrams may be interpreted as a physical model involving overlapping compartments. Still there is a need for corresponding tests for arguments structured by conditionals (if..., then...; ...only if...) and disjunctions (...or...). The proposal advanced here employs diagrams of a thermometer for arguments containing necessary or sufficient conditions.

The idea of using physical models to test validity occurs in the 3rd edition of Critical Reasoning by Cederblom and Paulsen,' but in the form of parallel upright domino-like blocks for conditionals, and curtained chambers for disjunctions. Unfortunately, these authors all but abandoned the idea in the 4 th edition ${ }^{2}$, seemingly due to difficulties in developing a satisfactory practical application. For instance,

1. Constructing diagrams of blocks or chambers requires a degree of artistic skill beyond the capacity of many students.

2. It is difficult to formulate specific, adequate instructions for conducting these tests, so they rely exclusively upon the explanation of a few illustrations.

3. The diagrams they construct include information from the conclusion as well as the premises - thereby vitiating the test. Their diagrams can never show how the premises might be true while the conclusion is false, for invalid arguments, because they are always shown containing the conclusion.

4. Application is restricted to sufficient conditions (using the "If..., then..." logical connective) while ignoring necessary conditions (...only if...).

Concerning this last point, perhaps the authors assume that every statement of a necessary condition may be converted into an equivalent statement of sufficient condition without significant loss or distortion of meaning. However, in practical discourse involving intentionality and/or events that might be causally related in either direction, this is highly problematic. For instance, the equivalent statement for "If NATO will bomb Serb forces, then Milosevic will attack the Kosovar Albanians" cannot be "NATO will bomb Serb forces only if Milosevic will attack the 
Kosovar Albanians." Appealing to the formal relationship:

If $\mathrm{A}$, then $\mathrm{B} \equiv \mathrm{A}$ only if $\mathrm{B}$

merely serves to obscure the obvious non-equivalence in their practical meaning: In the first instance, the attack is an intended response to the bombing; in the second, the bombing is an intended response to the attack. The point here is that necessary and sufficient conditions generally should be treated separately when reasoning within real life contexts. Inferences concerning how such statements are related should be drawn carefully for each specific case.

The approach proposed in this paper attempts to avoid the foregoing problems. In a manner similar to Venn diagrams, validity tests using a model of a thermometer is comprised of two steps:

1. We first attempt to diagram all-and solely - the information in the premises in such a way that the completed diagram will not contain the information expressed in the conclusion. This is analogous to the familiar method of trying to imagine a possible situation where the premises are true and the conclusion false.

2. We then determine the validity of the argument by inspecting the completed diagram to see whether it does express the information from the conclusion. If so, then the argument is valid; if not, then it is invalid, for we will have shown that even when the premises are true, the conclusion may still be false.

\section{Application of the Thermometer Test}

A statement that asserts either a sufficient condition or a necessary condition for something is analogous to the physical relation between two levels of mercury in a thermometer:

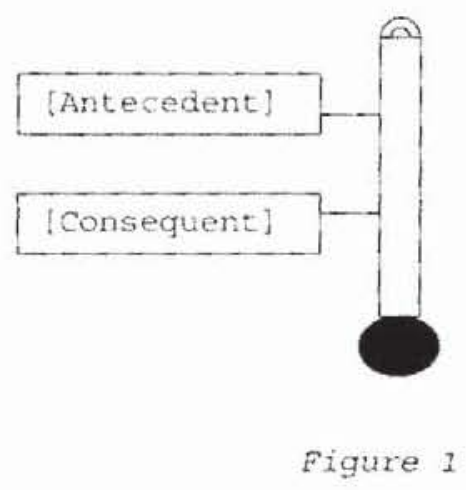

A sufficient condition is the antecedent of a statement of the form, "If [antecedent], then [consequent]." Whenever the mercury in the thermometer has reached the higher level, this is sufficient to know that it has already reached the lower level. A necessary condition is the consequent in a statement of the form, "[antecedent] only if [consequent]." It is necessary for the mercury in the thermometer to reach the lower level before it can reach the higher level. Thus, in either case, the antecedent is always situated above the consequent. Each conditional premise 
is represented by two such marks, whereas the actual level of mercury in the thermometer is determined relative to the condition that the nonconditional premise affirms or denies.

I will first illustrate the application of the thermometer method for testing the validity of conditional arguments having the simplest argument forms, and then describe the general procedure for employing it. Let us consider the following cases, beginning with the simplest ones that contain no negatives.

\section{Example 1 (Modus Ponens)}

If the Eiffel Tower is in Las Vegas, then it is in Nevada.

The Eiffel Tower is in Las Vegas.

$\therefore$ The Eiffel Tower is in Nevada.

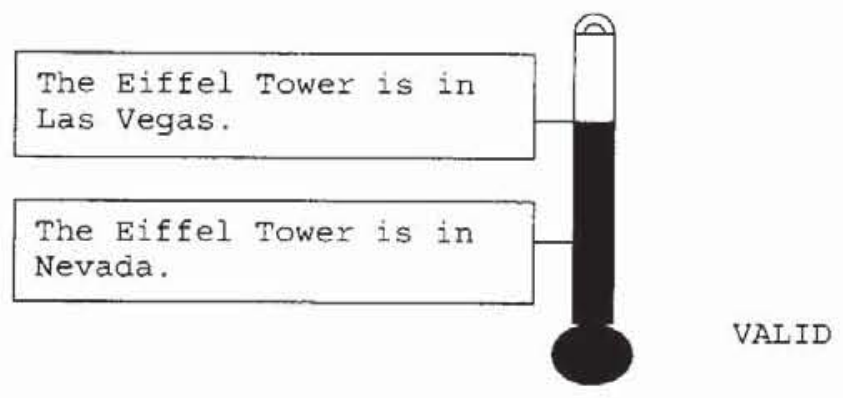

Figure 2

We first represent the conditional premise by setting up a thermometer having two marks corresponding to the relationship between the two conditions, the antecedent above the consequent. The conditional statement determines the position of those marks. In Figure 2 the mercury reaching the upper mark is sufficient for it to have reached the lower mark, so the upper mark stands for the antecedent, and the lower mark for the consequent. Label the mark corresponding to the antecedent with the information from the sufficient condition, "The Eiffel Tower is in Las Vegas." Label the mark corresponding to the consequent with the information from the other condition, "The Eiffel Tower is in Nevada."

Labeling in this manner has important advantages. It eliminates the need for symbolic translation (as would be required by the approach of propositional logic) in order to determine the validity of conditional arguments. The abstractions of formal logic are often unnecessarily burdensome and of limited utility in the praxis of reasoning critically. Simplifying the conditions has the advantage of avoiding much of the complexity arising from negation (for instance, when an antecedent or consequent includes the term not, or involves a prefix such as 'un-', 'in-', or 'dis-'). The thermometer method treats the entire antecedent and the entire consequent as the related conditions, and any nonconditional statement as a simple affirmation or denial of a condition.

A diagram must contain all of the information expressed in all of the premises 
of an argument, but nothing more. For arguments based on conditionals, that information is visually represented by the marks on the side of a thermometer and the level of mercury within it.

The conditional premises are used to position the marks next to the thermometer. Remember that "If..., then..." states a sufficient condition; "...only if..." states a necessary condition. In this example, "The Eiffel Tower is in Las Vegas" is the sufficient condition, so it is placed above the consequent. When the mercury is at the higher level, this is sufficient to know that it has already reached the lower level. The nonconditional premise determines where to set the level of mercury within the column. If it affirms a condition, such as, "The Eiffel Tower is in Las Vegas," this means that the level of mercury is set precisely at the corresponding mark. If it denies a condition, this means that the mercury has not risen to the corresponding mark, so it must be set somewhere below that level. In that case, we seek to position it at a level that would show when the argument is invalid.

The test relies on the definition of validity: An argument is valid when its premises cannot be true and its conclusion false (at the same time, and in the same respect); otherwise it is invalid. The diagram has been constructed to reflect what the premises state while trying to avoid conveying any information from the conclusion. Hence, we have only to inspect it to determine, on this ground, whether it also contains what the conclusion states. The conclusion, "The Eiffel Tower is in Nevada," is represented by finding the level of mercury at or above the corresponding mark, which is obviously the case here. This shows that these premises do lead inescapably to the conclusion, so the argument is valid.

\section{Example 2 (Affirming the Antecedent)}

Dogs are mammals only if they are vertebrates.

Dogs are mammals.

$\therefore$ Dogs are vertebrates.

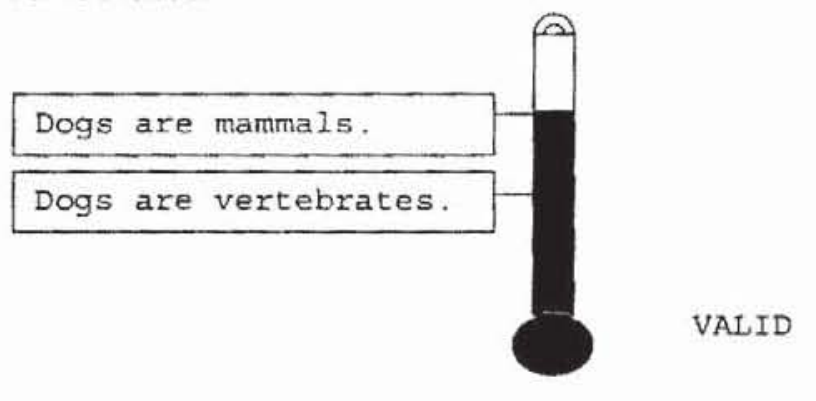

Figure 3

This diagram has the same essential structure as the previous one, even though the argument uses a conditional statement that focuses on a necessary, rather than a sufficient, condition. The test works for either form, so there is no need to 
translate conditional statements into the "If..., then..." form. By treating the argument in the form that is originally given, we maintain the advantage of simplicity while avoiding the danger of distorting meaning in problematic cases of determining an equivalent form. In general, a conditional that states a necessary condition in the consequent is true whenever the antecedent cannot be true without it. The conditional premise in this case claims that dogs' being vertebrates is a necessary condition for their being mammals, so the mark representing the necessary condition must be placed lower than the condition for which it is necessary. It is necessary for the mercury to pass a lower mark before can rise to any mark above it. Again, the nonconditional premise affirms the antecedent, which sets the level of mercury at the lower mark. The conclusion of the argument affirms the consequent, so the argument will be valid if the diagram shows the level of mercury at or above the lower mark. It does.

Example 3 (Denying the Sufficient Condition)

The Yugo is a reliable car only if its brakes work.

The Yugo is not reliable.

$\therefore$ The Yugo's brakes do not work.

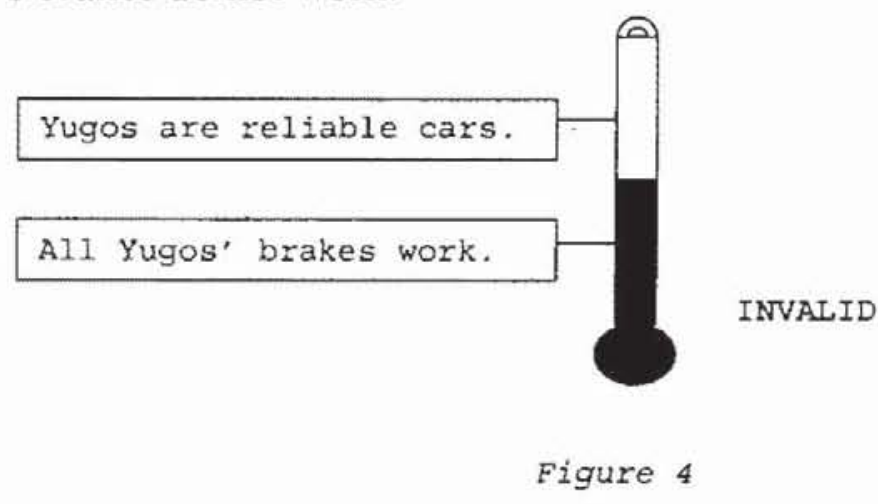

This example differs from Example 2 in that the nonconditional premise denies the sufficient condition (the antecedent). ${ }^{3}$ Whether the condition is positive or negative, denying it simply means making a statement that is contrary to, and thus incompatible with, what it claims. For our diagram, just as affirming a condition means that the mercury has reached the level of that condition, denying a condition means that the mercury has not reached that level.

When setting the level of mercury to correspond to a premise that affirms a condition, there is no possible variation-simply set it at the corresponding mark. In contrast, when setting the level for a premise that denies a condition, we must set it below the corresponding mark, also taking care to avoid including information from the conclusion, whenever possible. ${ }^{4}$ Whenever the conclusion affirms a condition, it is the same as claiming that the mercury is at or above the corresponding mark, so we try to set the level of mercury below that mark to test an argument's validity. Whenever the conclusion denies a condition, it is the same as 
claiming that the mercury is below that mark, so we try to set the level of mercury above that mark. This means that whenever there is some freedom in choosing where to set the level of mercury one must clearly keep in mind what the conclusion affirms or denies.

In this case, the conclusion denies the consequent, that the brakes do not work. Thus we place the level of mercury below the top mark, but above the bottom mark. We have been able to construct the diagram in such a manner as to exclude what the conclusion claims while granting the premises, so the argument is invalid.

Example 4 (Denying the Antecedent)

If AIDS is transmitted by kissing, then we should not kiss strangers.

AIDS is not transmitted by kissing.

$\therefore$ We should kiss strangers.

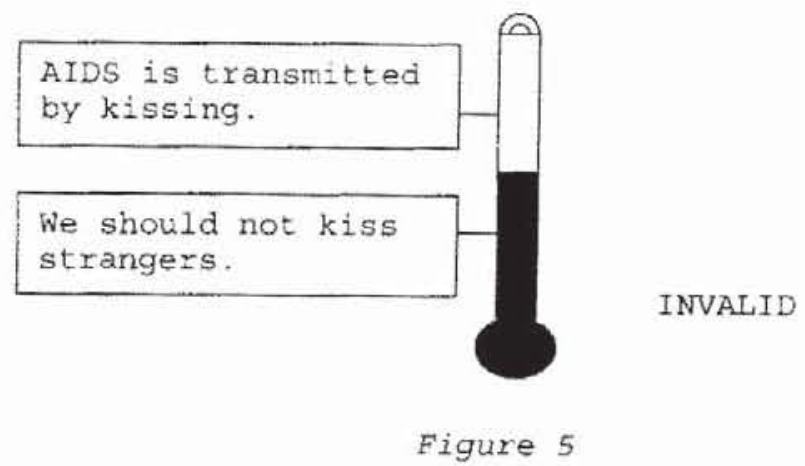

Even though it is grammatically possible to invert the order of an "If..., then..." statement, the antecedent always follows the 'if.' The second noteworthy feature that differs from the preceding examples is that one of the conditions, "We should not kiss strangers," is negative. The logical operator 'not' does not affect our procedure, although it calls for greater care and attention.

This example resembles the previous case insofar as the nonconditional premise also denies the sufficient condition, so the actual level of mercury must be drawn somewhere below the top mark. However, in deciding whether to place it above or below the lower mark, we must position it, if possible, to be incompatible with the conclusion. Because the conclusion denies the condition in the consequent, "We should not kiss strangers," it would correspond to any level of mercury below the lower mark. Thus, to show that the conclusion can be false, the actual level must be drawn above this mark. Since we have the freedom to do this here, it establishes that the argument is invalid. 


\section{Example 5 (Modus Tollens)}

If democracy works, then most people vote.

It is not the case that most people vote.

$\therefore$ Democracy does not work

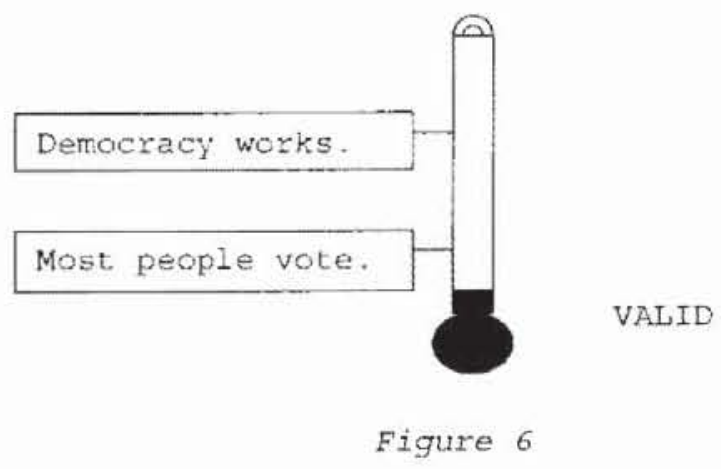

The nonconditional premise denies the claim that most people vote, so the mercury is set below that mark. This also represents the conclusion, "Democracy does not work," inasmuch as the level of mercury must be below that mark as well. Hence, the argument is valid.

\section{Example 6 (Denying the Consequent)}

Genetically altered food should be sold to the public only if it has been found safe in extensive tests on humans.

Genetically altered food has not been found safe in extensive human testing.

$\therefore$ Genetically altered food should not be sold to the public.

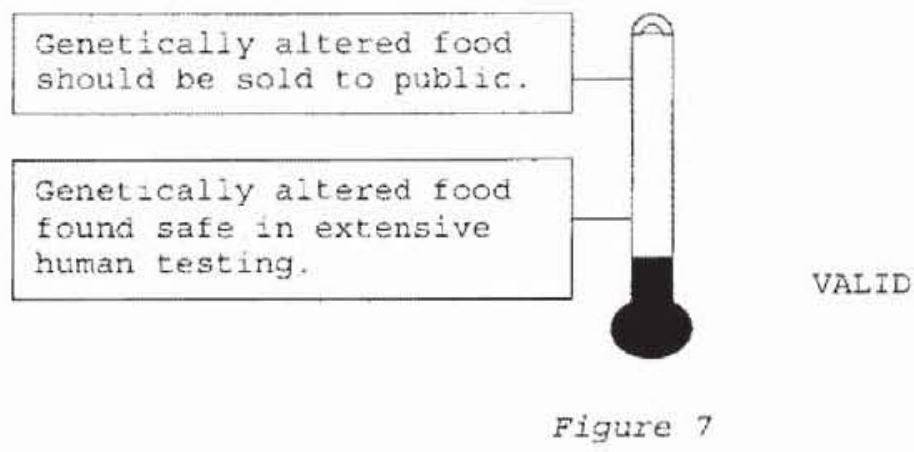

The necessary condition, genetically altered food having been found safe in extensive tests on humans, corresponds to the lower mark because it is necessary for the mercury to reach the lower mark in a thermometer before it can reach any higher mark. The nonconditional premise, that "[genetically altered food] has not been found safe in extensive tests on humans," denies the necessary condition. This is analogous to denying that the mercury has reached the lower mark, so the actual level in the thermometer is drawn below that mark. The diagram of this physical situation now accurately represents the premises. 
We now consider the conclusion. "Genetically altered food should not be sold to the public," denies the sufficient condition. This is analogous to denying that the mercury has reached the higher level. As we find the mercury below the corresponding mark, the diagram accurately depicts what the conclusion claims. We see that there is no way for the premises to be true and the conclusion false, so the argument is valid.

Example 7 (Affirming the Consequent)

If marbles are poisonous, then no one should eat them.

No one should eat marbles.

$\therefore$ Marbles are poisonous.

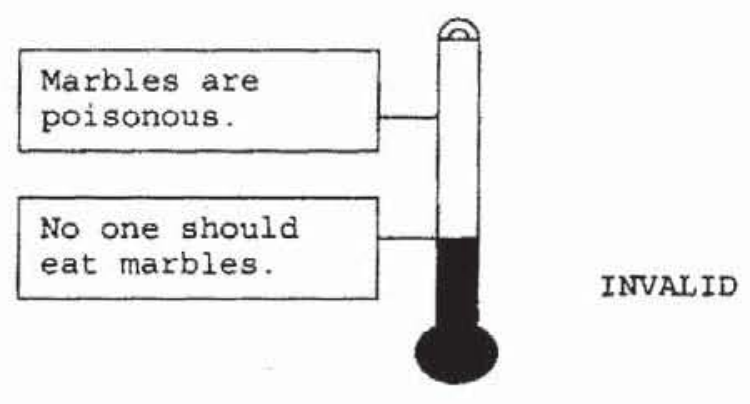

Figure 8

The diagram shows the level of mercury set at the lower mark, "No one should eat marbles." For the conclusion to be true, it would have to be at or above "Marbles are poisonous." Hence, the argument is invalid.

\section{Example 8 (Affirming the Necessary Condition)}

Ostriches can fly only if they have wings.

Ostriches have wings.

$\therefore$ Ostriches can fly.

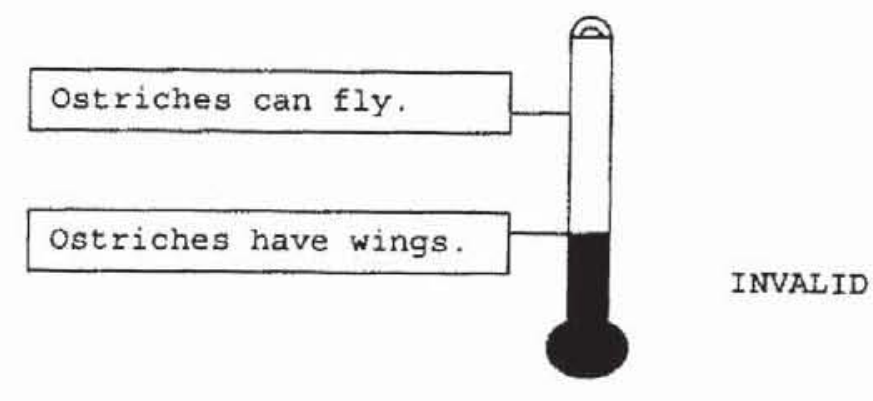

Figure 9 
Here the mercury is drawn at the level of the lower mark because the necessary condition is affirmed, rather than denied. As in the previous example, for the conclusion to be true, the mercury would have to be at or above the upper mark. Invalid.

For chains of conditionals, whenever there is more than one conditional statement in an argument, use a single thermometer in which any condition that is the same in more than one conditional premise is represented by a single mark. Great care must be used in constructing the diagram according to the principle of attempting to show how the premises may be true while the conclusion is false. Let us first consider arguments where all of the premises and the conclusion are conditional statements.

\section{Example 9 (Hypothetical Syllogism)}

If raptors had sharp teeth, then they were predators.

If raptors were predators, then they would have eaten small mammals.

$\therefore$ If raptors had sharp teeth, they would have eaten small mammals.

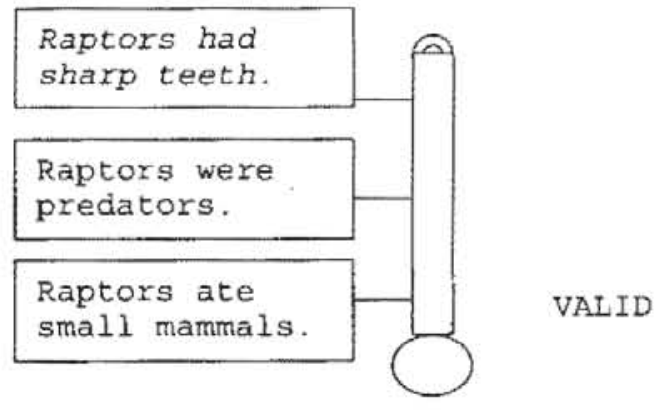

Figure 10

All of the premises of this argument are conditional, and they are linked to form a chain (the consequent of one gives a condition that is the antecedent of the other). ${ }^{5}$ The first premise in the chain is diagrammed with the antecedent above the consequent, as usual. The mark for the consequent now also serves to represent the antecedent of the second conditional, because it is the identical condition. Place the mark for the consequent of the second conditional below it to complete the diagram. No condition in the argument is either affirmed or denied, so there is no specified level of mercury in the column. It is drawn empty.

In this example the conclusion is also conditional. Its truth must therefore be considered hypothetically, that it, by inspecting the relationships between the marks to see whether the antecedent of the conclusion is above its consequent in the thermometer. "Raptors had sharp teeth" is the top mark, whereas "Raptors would have eaten small mammals" is the bottom mark, so the former is a sufficient condition for the latter. Since this is precisely what the conclusion states, the argument valid. 
Let us compare the previous case to one where a choice is necessary in deciding the order of the marks.

Example 10 (Defective Hypothetical Argument)

If Hoover Dam breaks, then the Colorado River will flood.

If Hoover Dam breaks, then the power grid will fail.

$\therefore$ If the power grid fails, then the Colorado River will flood.

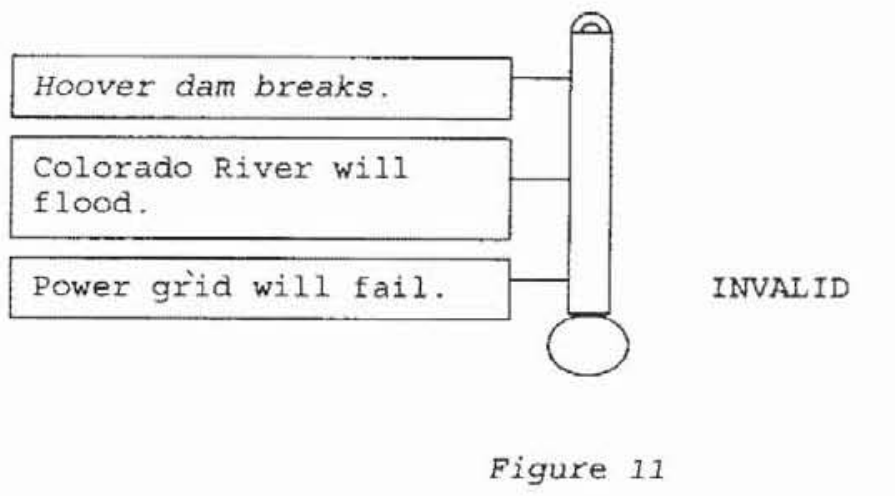

Because the antecedent for each of the premises is the same ("Hoover Dam breaks."), it does not matter which of them is diagrammed first. The antecedent of the other premise is simply represented by the mark already drawn, but its consequent will have to be placed relative to the first consequent such that it is contrary to the argument's conclusion, if possible. That is, "The power grid fails" must be located below "The Colorado River floods," which would show that the former is not a sufficient condition for the latter. The diagram expresses all the information in the premises without expressing the conclusion, so the premises do not entail the conclusion. Thus the argument is invalid.

Example $11 \quad$ (Principle of Hypothetical Syllogism)

A flat tax will be passed only if it is popular.

A flat tax will be popular only if it is fair.

$\therefore$ A flat tax will be passed only if it is fair.

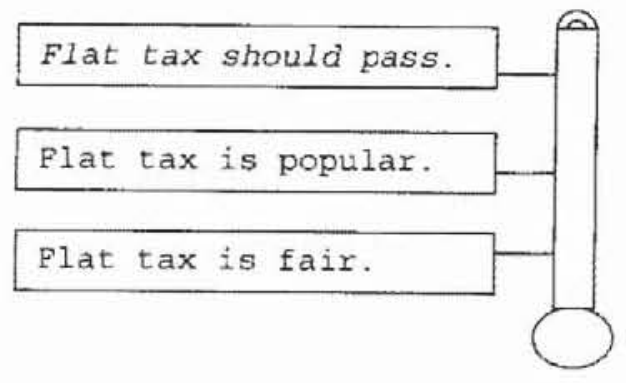

VALID 
Except for the labels, this diagram is the same as for Example 9. The premises state necessary conditions, so the necessary condition lies below the condition for which it is necessary. The condition, "A flat tax will be popular," occurs in both premises, so a single mark represents it in the diagram. The conclusion states that "A flat tax is fair" is a necessary condition for "A flat tax will be passed." As the diagram shows the former to lie below the latter, it is a necessary condition for it, so the conclusion logically follows from the premises. Valid argument.

\section{Example 12 (Invalid form of Hypothetical Argument)}

If raptors had sharp teeth, then they were predators.

If raptors were predators, then they would have eaten small mammals.

$\therefore$ If raptors ate small mammals, then they had sharp teeth.

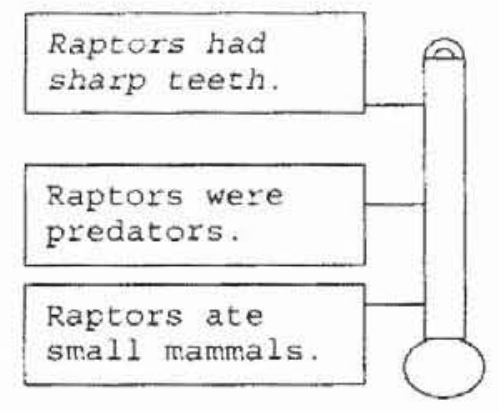

Figure 13
INVALID

The two linked conditional premises are diagrammed in the usual manner, with a single mark representing the common condition. In this case, when we test to see whether the diagram also shows the truth of the conclusion we find its antecedent, "Raptors ate small mammals," below its consequent, "Raptors had sharp teeth." The antecedent therefore is not a sufficient condition for the consequent as claimed, so the conclusion is not represented in the diagram. As a result, the argument is invalid.

Example 13 (Invalid form of Hypothetical)

Athletes use steroids only if they enhance athletic performance.

Steroids enhance athletic performance only if they make an athlete stronger or faster.

$\therefore$ Steroids make an athlete stronger or faster only if athletes use steroids. 


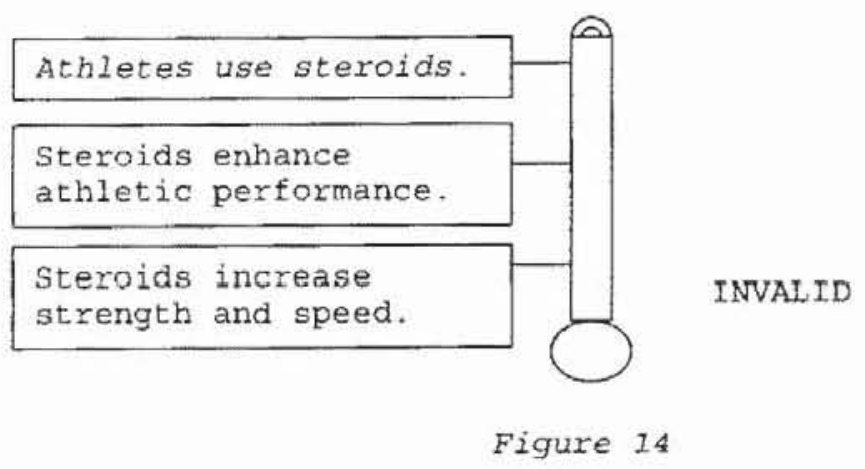

After diagramming the premises, we find that it shows the consequent of the conclusion above its antecedent, which means that it cannot be a necessary condition for it. Consequently, the diagram does not reflect what the conclusion states, which shows that this argument is invalid.

\section{Example 14 (Chain Argument)}

If raptors had sharp teeth, then they were predators.

If raptors were predators, then they ate small mammals.

Raptors had sharp teeth.

$\therefore$ Raptors ate small mammals.

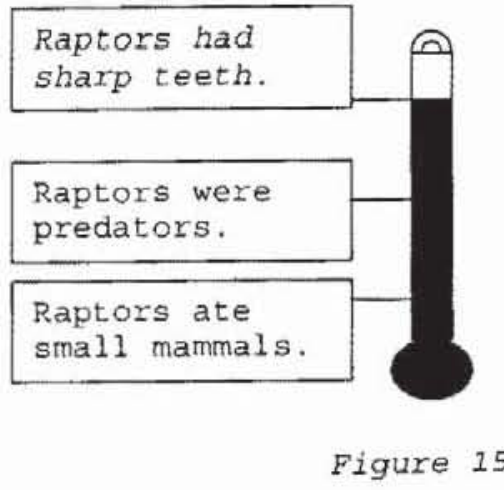

In principle, we may extend the application of physical models to conditional premises that are linked, no matter the length of the chain. This argument form may be understood as relying on successive applications of the principle of Modus Ponens. It differs from Hypothetical Syllogism in that there is a nonconditional premise that allows us to set the level of mercury. Here the condition, "Raptors were predators," occurs in both conditional premises, so it is represented on the thermometer by a single mark. The nonconditional premise affirms the first sufficient condition, "Raptors had sharp teeth," thus setting the level of mercury at the top mark. The conclusion, "Raptors ate small mammals," now must be contained within the diagram as well, for the mercury already has exceeded this mark. Consequently, valid argument.

Consider a variation of this argument where the premise "Raptors had sharp teeth," was missing. Whenever the conclusion of such an argument is not condi- 
tional, its truth is indeterminate, and thus may be false, insofar as there is no nonconditional premise to set the level of mercury anywhere. Thus we must always judge it, and any argument of this sort, to be invalid. However, its defect is so obvious that we are obliged by the Principle of Charity to assume that the author intended to affirm the antecedent of the first conditional in the chain, which would make the argument valid.

\section{Example 15 (Chain Argument)}

The U.S. is the world's sole superpower only if it has vastly greater military capability than any other country.

The U.S. has vastly greater military capability only if its military budget is more than double that of any other country.

The U.S. is presently the only superpower in the world.

$\therefore$ The U.S. is presently spending more than twice what any other country spends on its military.

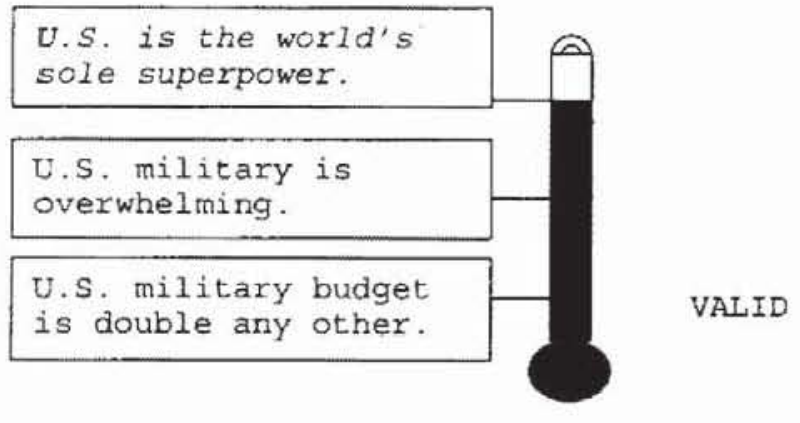

Figure 16

A chain of necessary conditions works much the same way as for sufficient conditions. After diagramming the conditional premises, set the level of mercury at the mark corresponding to the condition affirmed by the nonconditional premise, in this case, "The U.S. is the world's sole superpower." Because the level of mercury has passed the mark corresponding to the conclusion, the diagram represents what the conclusion states. Thus the argument is valid.

What if the conclusion were switched with the nonconditional premise? In that case the mercury would have been set at the lower mark, but the conclusion would require it to be at or above the top mark. That version of the argument would be invalid.

Example 16 (Invalid form of chain argument)

If professional wrestling is a legitimate competition, then the winners are not decided in advance.

If winners are not decided in advance, then all injuries are real.

Pro wrestling is not a legitimate competition.

$\therefore$ Not all injuries are real. 


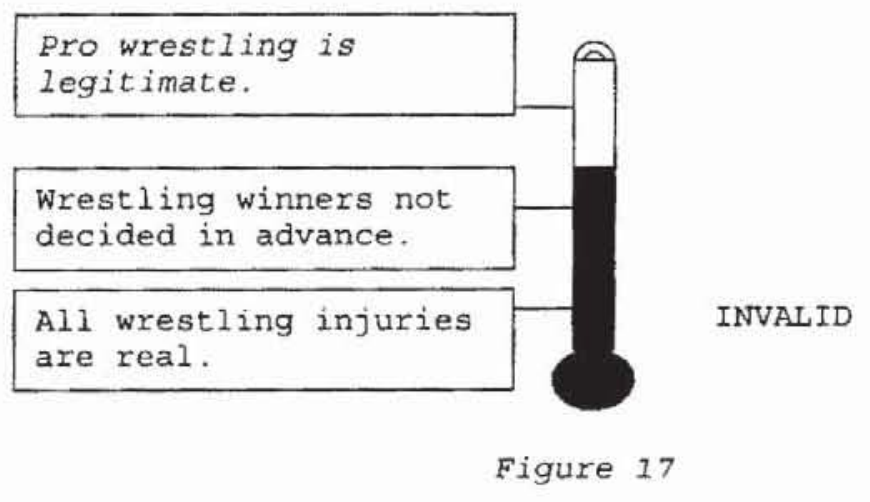

This argument uses the fallacious principle of Denying the Antecedent within the context of a chain of conditionals. The mercury is set below the "Pro wrestling is legitimate" mark, but above the "All injuries are real" mark to show that the conclusion can be false.

\section{Example 17}

If yellow-green is the brightest color then it is the most visible.

If yellow-green is the most visible, then all public vehicles should be painted that color.

Not all public vehicles should be painted yellow-green.

$\therefore$ Yellow-green is not the brightest color.

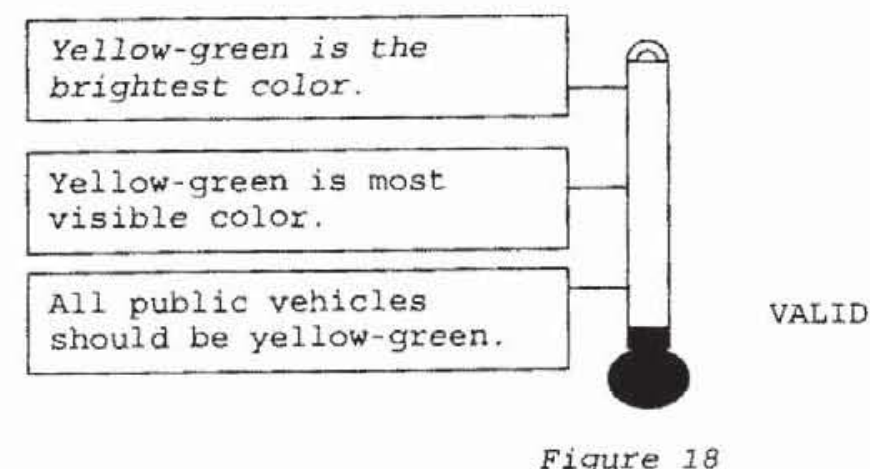

Figure 18

This argument uses the principle of Modus Tollens within a chain of conditionals.

Example 18 (Hypothetical Argument using Mixed Conditionals)

If the Republicans win the Senate then they win the House.

The Republicans will win the House only if they win Texas.

$\therefore$ If the Republicans win Texas, then they will win the Senate. 


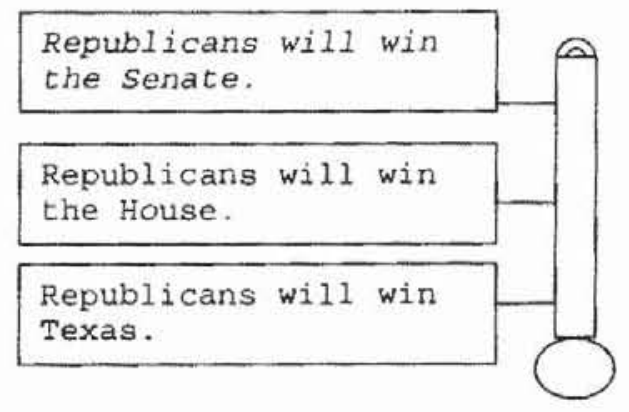

INVALID

Figure 19

This example illustrates a combination of premises stating necessary and sufficient conditions. Again, the common condition is represented by a single mark. In . this case, it does not matter which premise is diagrammed first. In diagramming the premise stating the sufficient condition, position it (The Republicans will win the Senate.) above the consequent (The Republicans will win the House.). For the premise stating the necessary condition, position it (The Republicans will win Texas.) below its antecedent (The Republicans will win the House.). According to the completed diagram, the conclusion fails to be expressed for the reason that the sufficient condition that it states (The Republicans will win Texas.) lies below "The Republicans will win the Senate." This shows the argument to be invalid.

\section{Example 19 (Chain Argument using Mixed Conditionals)}

Congress should cut taxes only if there is a surplus.

If there is a surplus, then the national debt is decreasing.

The national debt is still increasing.

$\therefore$ Congress should not cut taxes.

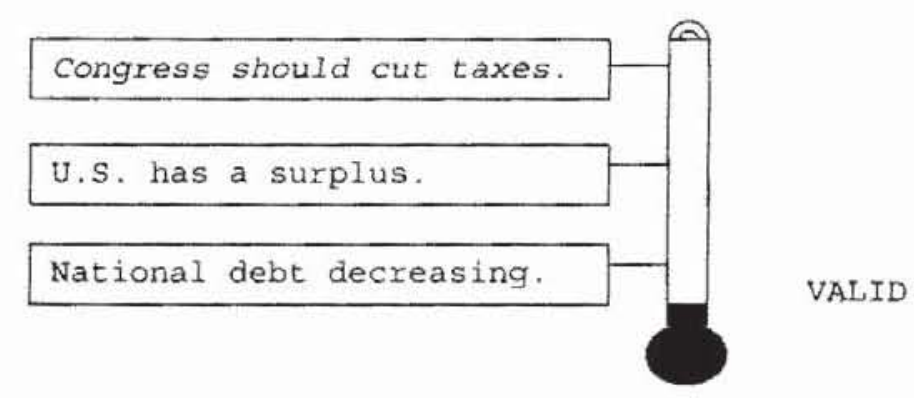

Figure 20

In this case too, either the necessary or sufficient condition may be diagrammed first, with the common condition designated by the same mark. As the necessary condition, "There is a surplus" will be located under the "Congress should cut taxes" mark; as the sufficient condition, "There is a surplus" will be located above the "The national debt is decreasing" mark. Because increasing is the opposite of decreasing, the nonconditional premise denies the condition stated in the lower 
mark, so the mercury will be drawn somewhere below it. The conclusion, "Congress should not cut taxes," denies the condition at the top mark. The diagram expresses what the conclusion states because the level of mercury below this mark, so the argument is valid.

\section{Example 20 (Invalid Chain of Conditionals)}

If raptors had sharp teeth, then they were predators.

If raptors were predators, then they would have eaten small mammals.

Raptors ate small mammals.

$\therefore$ Raptors had sharp teeth.

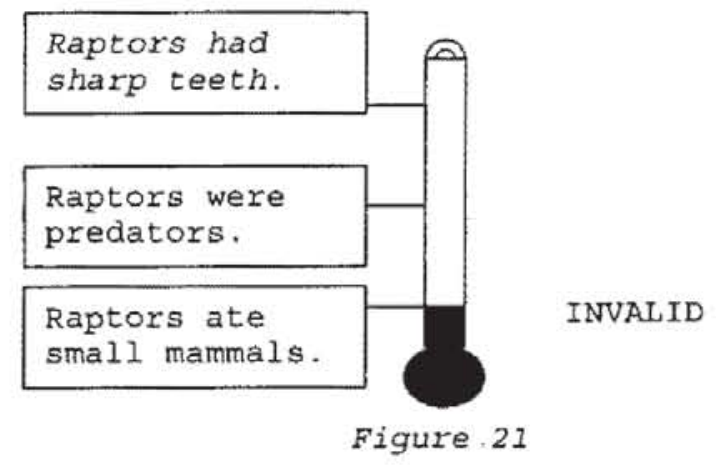

Here we find the fallacious principle of Affirming the Consequent applied to a chain of conditionals. This is invalid.

\section{Example 21}

If the $\mathrm{Y} 2 \mathrm{~K}$ problem causes widespread disruption, then corporate profits will decline.

If corporate profits decline, then the U. S. stock market will crash.

Few people believe that the stock market will crash.

$\therefore$ The $\mathrm{Y} 2 \mathrm{~K}$ problem will not cause widespread disruption.

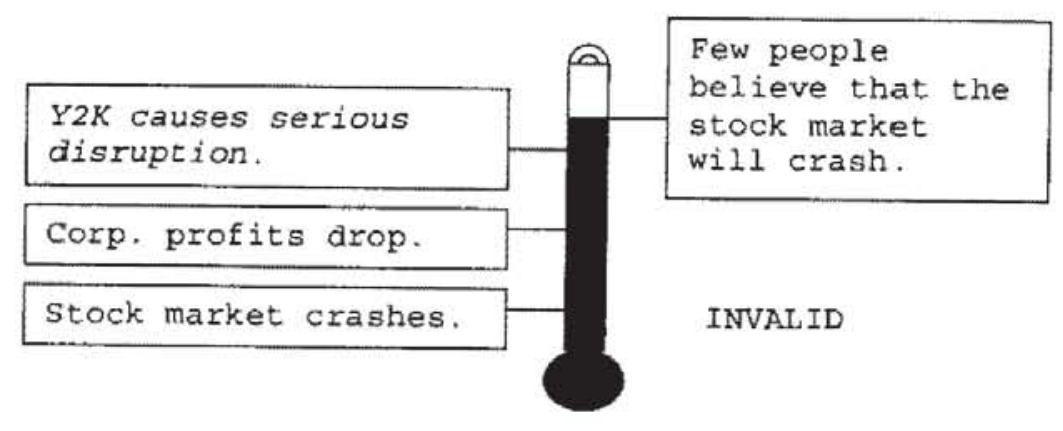


This example also illustrates linked conditionals, but the nonconditional premise, "Few people believe that the stock market will crash," refers only to popular belief about the issue. Thus it neither affirms nor denies any of the conditions contained in the conditional premises. The claim, "Few people believe that the stock market will crash," is neither equivalent to, nor does it logically imply, "The stock market will not crash." This means that it requires a separate mark on the thermometer, which can always be placed in such a position that would prevent the diagram from expressing what the conclusion states. Here we place it above the top mark to show that "The Y2K problem does not cause widespread disruption" can be false. Consequently, the argument is invalid.

\section{General Description of the Thermometer Test}

From these examples it should now be possible to establish general rules for applying the thermometer test. Conditionals are used to set marks against which to measure the level of mercury in a thermometer. Affirmation or denial in a premise that is a simple nonconditional statement establishes its actual level in the column. An affirmation is represented as if true whenever the mercury appears at or above the corresponding mark; otherwise it is represented as if false. A denial is represented as if true whenever the mercury appears below the corresponding markotherwise it is represented as if false.

1. Every conditional premise in an argument is diagrammed by placing a pair of marks (antecedent above consequent) alongside a single thermometer. Whenever the same condition occurs in more than one conditional premise, whether it occurs as an antecedent or as a consequent, it is represented by a single mark (Examples 9 to 22). Each mark is labeled to correspond to the condition stated in the complete antecedent or consequent (whether positive or negative) from which it was derived.

2. Whenever a nonconditional premise affirms a particular antecedent or consequent, draw the actual level of mercury exactly at the corresponding mark (Examples 1,2,7,8,14,15,20).

3. Whenever the nonconditional premise denies a particular antecedent or consequent, draw the level of mercury below the corresponding mark, but at a location that would be inconsistent with the conclusion, if possible (Examples $3,4,5,6,16,17,19)$.

4. Whenever the nonconditional premise corresponds neither to an antecedent nor to a consequent, set the level of mercury at a mark drawn at a location that would be inconsistent with the conclusion (Example 2I).

\section{Method of Determining Validity}

We diagram all, but only, the information in the premises. We test for validity by mentally inspecting the resulting diagram to see whether it depicts the information stated in the conclusion. For an argument to be valid, it must be impossible for all 
the premises to be true and the conclusion false. By analogy, it must be impossible to diagram all of the information from all the premises without at the same time having the diagram include all the information contained in the conclusion. If it is indeed impossible, then the argument is valid; when it is possible to do this, the diagram shows the argument to be invalid.

\section{Advantages}

This method for testing validity has several advantages:

1. It provides a reliable procedure for determining the validity of simple conditional arguments.

2. Students need not comprehend the subject matter of the argument. Lack of background knowledge sometimes limits the test for validity that uses counterexamples. Where subject matter is technical or obscure, students may have little idea what it would mean for the premises or conclusion to be true or false.

3. The diagrams are easily drawn.

4. The method's simplicity allows anyone to use it. In particular, it can accommodate both "If..., then..." and "...only if..." logical connectives without any need for translation. It also applies to any condition, positive or negative, without any need for employing "not" as a logical operator (Examples 4 and 16).

5. When introduced to the logic of conditional arguments, some students will prefer visualizing models to procedures that require abstract formalism.

6. Insofar as these physical models are analogous to logical forms, working with them will aid the student in grasping the general logical forms underlying arguments having diverse content. Thus they can facilitate the gradual introduction of formalism.

\section{Endnotes}

'Jerry Cederblom and David Paulsen, Critical Reasoning, 3rd ed., (Belmont: Wadsworth, 1991), 68-75.

${ }^{2}$ Ibid., 95-96.

${ }^{3}$ Unreliable' simply means not reliable.

${ }^{4}$ This explicit consideration of the conclusion for the purpose of drawing an accurate diagram contrasts sharply with the mechanical technique of drawing Venn diagrams to test categorical arguments, which almost never presents us with options.

'More lengthy chains of conditionals introduces a limitation of this method for testing validity. It requires that we introduce a rule that we shall not regard an argument as valid if there is any link missing in the chain. This might be remedied if an implicit premise is added to complete the chain. However, whether this may be done as part of the reconstruction process depends on applying principles and guidelines for reconstructing arguments whose merit we have insufficient scope to discuss.

${ }^{6}$ The conclusion is not "All wrestling injuries are not real," for that is not the contradictory of the original conclusion, and so would not match the given logical form. 